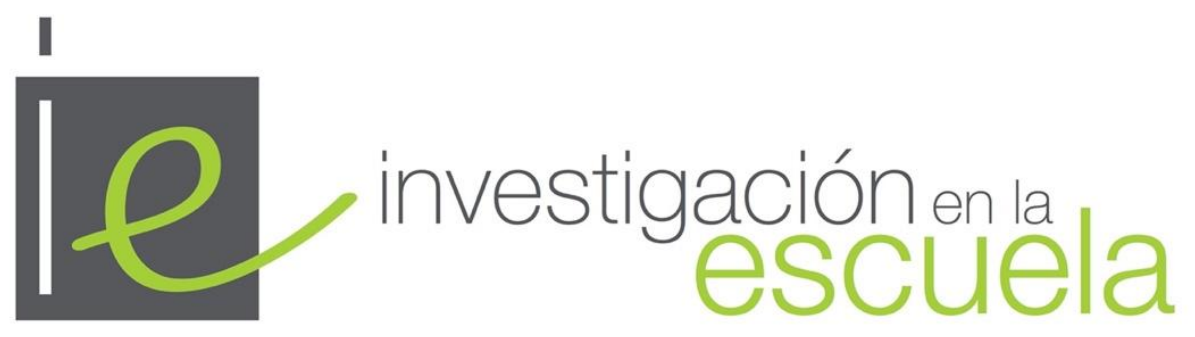

Revista de Investigación e Innovación Educativa nº 101, 2020 | e-ISSN 2443-9991

\title{
La escritura reflexiva como mediación en la formación inicial de docentes. Pensar la enseñanza, inscribir la experiencia
}

\author{
Reflective writing as a mediation in teacher education. Thinking about teaching, enrolling experience
}

iD Mgtr. Silvia Laura Muñoz es Profesora Adjunta en la Facultad de Ciencias Humanas de la Universidad Nacional de San Luis (Argentina)·silvialmunoz@gmail.com https://orcid.org/0000-0003-3076-5816

iD Graduada Laura Pañagua Domínguez es Personal Investigador en Formación en la Facultad de Educación de la Universidad de Málaga (España)·laurapani76@uma.es https://orcid.org/0000-0003-2888-6651

iD Dra. Nieves Blanco García es Profesora Catedrática en la Facultad de Educación de la Universidad de Málaga (España)·nblanco@uma.es https://orcid.org/0000-0001-7735-4593

\begin{abstract}
Resumen. En la formación inicial de docentes, la escritura puede constituir una mediación fructífera para promover una relación pensante con el propio hacer y para desarrollar un saber pedagógico arraigado en la experiencia de quien se forma. Este artículo presenta una propuesta desarrollada con estudiantes practicantes, en la cual se asigna un lugar fundamental a la escritura en relación con la práctica y la propia experiencia formativa. En él exponemos las concepciones que sostienen nuestra acción docente, algunos de los recursos narrativos utilizados y analizamos textos elaborados por estudiantes en este contexto. Hemos constatado que la escritura personal-reflexiva crea un espacio de exploración de sí, del propio ser y hacer como estudiante en relación al oficio de enseñar, y posibilita desarrollar una relación narrativa con lo vivido en la formación, conectando la historia personal con la identidad y el saber docente en construcción.
\end{abstract}

Abstract. In teachers' education, writing can be a valuable mediation to promote a thinking relationship with one's practice and to develop professional knowledge linked to one's personal experience. This article explores a proposal developed with student teachers, in which writing has an essential place in relation to teaching practice and one's formative experience. We present the concepts underlying this proposal, some narrative resources that compose it and also analyze texts produced by students in this context. We found that reflective writing creates a space of inquiry about oneself as student teacher and promotes a narrative relationship, linking the experience of being a student teacher to one's personal history and to the building of professional identity and knowledge.

\section{Palabras clave $\cdot$ Keywords}

Formación de profesores, escritura, práctica pedagógica, experiencia pedagógica, enseñanza, profesor, estudio de casos, investigación.

Teacher education, writing, teaching practice, teaching experience, teaching, teacher, case study, research.

\section{Introducción}

La docencia es una tarea compleja que requiere el dominio de conocimiento de diversos tipos; no obstante, el saber pedagógico que este oficio reclama es un saber "habitado", ligado a la experiencia, a la práctica y a lo vivido, que nos permite decidir qué es lo adecuado en cada situación concreta (van Manen, 2015). Consecuentemente, la formación no puede pensarse como acumulación de conocimientos para aplicarlos luego, sino que requiere atender a los modos de pensar y de actuar que quienes se forman maduran y despliegan, a partir de las experiencias que viven y los conocimientos y recursos con los que se relacionan. 
Una forma de acompañar este proceso puede concretarse a través de la escritura entendida como una mediación, "un modo de territorializar la experiencia vivida, ponerle nombre a ese devenir profesor/a, marcar una frontera, limitar lo vivido en unos trazos y unas huellas que dan cuenta de cómo cada sujeto atravesó la experiencia de la formación" (Sardi, 2013, p. 14). En este sentido, dice Souto (2016), al narrar la experiencia "el sujeto que la vive se incluye, se afecta, se implica y desde su subjetividad la hace inteligible" (p. 56).

En este artículo exploramos la escritura como mediación en la formación, a partir de la propuesta formativa que una de las autoras desarrolla con estudiantes del último año del Profesorado Universitario en Letras (Universidad Nacional de San Luis, Argentina) en la asignatura Práctica Docente en Lengua y Literatura. En esta propuesta, la escritura tiene un papel fundamental y se desarrolla a través de una variedad de recursos narrativos, poniendo énfasis en aquellos que articulan la narración de las propias vivencias y hacer en las prácticas, como fuente del desarrollo de la propia identidad y de saber profesional.

\section{La formación y el desarrollo de saberes profesionales}

La educación es una tarea delicada, abierta al riesgo de lo imprevisible (Biesta, 2017), que nos requiere en presencia viva y en donde nuestra subjetividad se pone en juego. En la actualidad, hay un amplio reconocimiento de que el saber necesario para la enseñanza implica a la totalidad de la persona, que está vinculado a la práctica y configurado por una amalgama de componentes cognitivos y emocionales, intelectuales e intuitivos, conscientes y experienciales (Tardiff, 2004; Pérez Gómez, 2010; Alliaud, 2017). Esto implica que los saberes docentes no se reducen a un conocimiento técnico y disciplinar, sino que están ligados a la vida y a la propia experiencia, reconocida y reelaborada (Contreras, 2011; 2016).

Al pensar en el conocimiento necesario para la enseñanza, Connelly y Clandinin (1988) señalan que, aunque la distinción teoría-práctica está muy asentada en la tradición pedagógica, resulta de más interés la conexión entre experiencia-saber porque lo que la enseñanza requiere no es tanto aprender algo nuevo (lo que podríamos llamar teoría) cuanto "habitar" el conocimiento, incorporando eso nuevo a nuestra experiencia. Coincidentemente, van Manen (2015) plantea que la práctica de la enseñanza no debe entenderse como un campo de aplicación de conocimiento (teórico-académico), pues la acción docente no se sustenta en proposiciones sino en otras acciones previas, esto es, en la experiencia. También Cifali (2005) señala que los saberes constituidos son importantes en tanto que "puntos de referencia a la hora de interrogarnos sobre lo vivo en cada situación" (p.180), pero que los saberes docentes son saberes incorporados, personalizados y situados, fruto de una transacción constante entre lo que se es (incluyendo lo que se conoce, lo que se siente, lo que se espera) y lo que se hace. Como afirma Tardiff (2004), los profesores no solo piensan con la cabeza sino con la vida, con su propia vida y a partir de ella; por lo tanto, el profesor no es un sujeto epistémico sino un sujeto experiencial.

Prepararse para ser docente requiere que se practique un pensar detenido sobre las experiencias vividas y la oportunidad de activar la disciplina mental de reflexionar sobre las vivencias en las prácticas (Mortari, 2012). La disposición reflexiva se aprende, consecuentemente es necesario que las y los docentes en formación tengan experiencias formativas que expresamente les propongan reflexionar sobre el sentido de lo que hacen y de lo que sucede en las aulas. Y que tengan un acompañamiento que les permita analizar la realidad educativa y su intervención en ella, comprendiendo su sentido pedagógico y cuestionando los supuestos dados (Sierra, Caparrós, Molina y Blanco, 2017; Pañagua, Martín Alonso y Blanco, 2019; Contreras, Quiles y Paredes, 2019).

Desde esta perspectiva, el desafío que se plantea a quienes acompañamos los procesos formativos es crear condiciones y mediaciones que abran a vivir la formación como experiencia de "aprender a actuar en primera persona" (Sofías, 2007), tomando conciencia del propio ser pedagógico y asumiendo la responsabilidad de las propias acciones. En este sentido, consideramos que la escritura como mediación en la formación puede constituir una oportunidad para activar la disciplina de reflexionar sobre las propias vivencias de las prácticas -las representaciones, saberes y supuestos que están en juego en ellas- enlazando la experiencia de formación con la propia biografía educativa y proyecto profesional; porque como señalan Contreras y Pérez de Lara (2010), "la escritura es pasaje, puente, mediación, traducción entre vivir y pensar" (p. 81), donde la cuestión no es si (la escritura) dice la experiencia sino la posibilidad de imaginar, de abrir sentidos y dimensiones que inaugura. 


\section{La escritura como mediación en la formación inicial}

La escritura constituye un medio fundamental para dar forma al pensamiento, para reconstruir lo vivido y, así, comunicar algo de su cualidad y sentido, moviendo a la reflexión sobre la propia práctica y poniendo en tensión la propia biografía y expectativas de quien escribe, sus saberes disciplinares, representaciones acerca de la escuela y los sujetos que la habitan, las relaciones ante lo supuesto y lo actuado (Blake, 2013).

Escribir sobre lo que nos pasa puede ser una experiencia de formación (Sardi, 2013); pero no vale cualquier escritura. No sirve la que habitualmente aprendemos en el contexto escolar y continuamos en la universidad, centrada en la repetición y la reproducción sin arriesgar sentido propio (Carlino, 2005), limitada a expresar y ordenar ideas, soslayando su potencial de ir construyéndolas al explorar el sentido de lo que vivimos y pensamos. Al respecto, Alvarado y Cortés (2001), Bombini (2012), Serrano (2014) y Sardi (2013, 2017) -entre otros- han señalado que la escritura tiene un alto valor epistémico, en tanto promueve procesos de objetivación y distanciamiento respecto al propio discurso, destacando que ese descentramiento permite la revisión crítica y la transformación de las propias ideas, genera conocimiento y también la estructuración del pensamiento.

Asimismo, el tipo de escritura que requiere la formación es una escritura personal-reflexiva, que aspira a cultivar una relación fructífera entre experiencia y saber (Contreras, 2013; Contreras, Quiles y Paredes, 2019) al involucrar a quien escribe de modo auténtico, desde su historia personal y haciéndose presente con su subjetividad (Clandinin, 2013; Delory-Momberger, 2014; Souto, 2016), sus afectos, las dificultades que experimenta y las limitaciones que vive (Molina, Blanco y Arbiol, 2016, p. 113). Desde esta perspectiva, una escritura es valiosa en la medida que hace pasaje de la vivencia a la experiencia, pone al sujeto frente a esta última y "en ese mirarse y reflexionar sobre sí mismo y sus prácticas permite penetrar más allá del significado latente (...) e interpretar lo subyacente, lo no visible” (Sardi, 2013, p. 13). En síntesis, la escritura es personal y reflexiva cuando se practica como exploración de sí, más allá del subjetivismo simple de hablar de sí para adentrarse en el hablar desde sí -manteniendo la propia presencia como parte del relato- y, al ir buscando el sentido de lo que ha sucedido, cómo eso le afecta, qué le significa, pone "lo vivido en relación con lo que nos hace pensar, lo sentido con el intento de captarlo, lo pasado con lo que nos sentimos llamados a hacer" (Contreras, 2013, p. 14). Precisamente, lo que otorga a la escritura un valor formativo es escribir en primera persona, exponiéndose, para dejarse transformar en ese proceso, en el relato de una situación, de una vivencia (Cifali, 2010).

En la formación, escribir en clave personal y reflexiva es un aprendizaje importante para cultivar la disposición a mantener una relación pensante con la realidad (Mortari, 2012), una relación reflexiva que busca dar cuenta de lo vivido mirando más allá de lo conocido, de lo ya sabido, para abrirse a nuevas preguntas y sentidos. Este tipo de escritura resulta necesaria para ir alcanzando una conciencia más elaborada del propio pensamiento pedagógico, que va tomando forma a través de ella: los sucesos van ordenándose, se producen asociaciones, se tejen nuevos vínculos, emergen recuerdos, preguntas, incertidumbres y, también, el no-saber... De igual modo, es una oportunidad para explorar y practicar un lenguaje sensible, ligado a la vivencia, que a través de la narración permite pensar sobre esa situación concreta y sobre las cualidades pedagógicas que podemos percibir en ella.

\section{Metodología}

El propósito de este artículo es analizar el potencial de la escritura personal-reflexiva como mediación en la formación, esto es, como espacio de indagación y aprendizaje que posibilita construir saber profesional arraigado en la propia experiencia formativa. Nos interesa explorar si la escritura que proponemos: (1) conecta el ser estudiante-docente en formación con el saber que el oficio educativo (les) requiere en las prácticas; (2) propicia una relación pensante con el propio hacer docente; y (3) moviliza la construcción de la propia identidad y saber profesional, al afectar los modos de pensar(se), entender(se) y actuar pedagógicamente.

Para ello, realizamos una lectura detenida de los diarios de formación, los guiones conjeturales y los autorregistros elaborados por 17 estudiantes que componen el grupo total que cursó la asignatura Práctica Docente en Lengua y Literatura en 2018; y un análisis más detallado de los escritos de tres integrantes del mismo, seleccionados atendiendo a dos criterios: el involucramiento personal en la escritura -evidenciado en la frecuencia, extensión y detalle de sus escritos-, y la profundidad de la escritura, considerando que trascienden el plano descriptivo de vivencias y acciones, a través de interrogantes y reflexiones vinculadas a la propia historia y proyecto de formación y al sentido pedagógico de sus prácticas. Nos preguntamos: ¿qué (nos) cuentan al escribir acerca de su experiencia formativa en las prácticas?, ¿de qué (nos) hablan?, ¿qué 
registran acerca de lo que (les) pasa en este espacio-tiempo de formación que compartimos?, ¿cómo se piensan en tanto docentes en formación?, ¿qué saberes ponen en juego al pensar la enseñanza y pensarse enseñando?

A continuación, presentamos la propuesta formativa de esta asignatura y, seguidamente, el análisis del material seleccionado, organizado en dos momentos: en el primero, damos cuenta del modo en que escribir en clave personal y reflexiva abre una relación narrativa con lo que cada una vive en este momento de su formación. En el segundo, abordamos la relación con el saber a modo de ejemplo de las inquietudes que se van abriendo a través de la exploración de sí que la escritura promueve.

\subsection{La propuesta formativa. Escribir (en) las prácticas}

La asignatura se desarrolla con modalidad de taller con clases teórico-prácticas, es de carácter anual, e implica una aproximación progresiva a la tarea docente en escuelas secundarias, donde cada estudiante se hace cargo de las clases de un curso durante un mes, y acompaña a un/a compañero/a en carácter de pareja pedagógica a lo largo de sus prácticas. Partimos de considerar que "saber es algo que nos pasa, no por acumulación de conocimientos, sino por la transformación que se produce en nosotros: es lo que nos pasa como producto de lo vivido hecho experiencia" (Alliaud, 2017, p. 74). Precisamente, una cuestión que tensiona nuestro proceder como formadoras es la relación con el saber que las y los estudiantes traen y ponen en juego en este espacio, considerando que año tras año la formación que esperan involucra una búsqueda, aparentemente desligada de sí y de sus biografías educativas, vinculada a representaciones construidas en la vivencia de lo escolar y a aspectos instrumentales de la enseñanza que esperan resolver.

En la propuesta, la escritura tiene un papel fundamental como mediación en las prácticas, dado su valor epistémico -en tanto posibilita construir conocimiento a partir de aquello que se escribe- y, sobre todo, buscando desplegar su valor formativo (Souto, 2016). De ahí que está vinculada tanto al quehacer docente en el aula como a la reflexión y exploración de las propias ideas y actuaciones pedagógicas, configurando un espacio de "indagación de sî" que implica la "búsqueda y comprensión del sentido con el que se viven las cosas, de la forma como nuestro pensamiento está moldeado por lo vivido” (Contreras, 2013, p. 14). Desde esta perspectiva, elaboramos un plan de trabajos prácticos incorporando recursos narrativos que son desarrollados a lo largo del año de manera articulada y recursiva. En este artículo focalizamos nuestro análisis en tres de ellos:

- El diario de formación, cuya escritura inician al comenzar la asignatura, registrando lo que pasa y les pasa en el transcurrir del curso, tanto en la universidad como en la escuela donde se integran para hacer sus prácticas. A través de su escritura buscamos hacer lugar a la experiencia atendiendo a cómo lo que van viviendo les afecta y abre a pensar y habitar la docencia, conectando con las propias historias y vivencias de la escuela y también, con lo que las lecturas y las clases que compartimos en la universidad les provoca. Así, van explorando los propios modos de aprender, de relacionarse con el saber, de enseñar y asumir(se) en la propia formación, como algo inacabado.

- El guion conjetural (Bombini, 2012), texto que desarrollan al momento de pensar la enseñanza e intervenir en el aula donde realizan sus prácticas. En el guion, cada estudiante-practicante planifica sus clases narrativamente, esto es, escribiendo en primera persona al imaginarse enseñando los contenidos que les asignan o eligen como temas a enseñar, argumentando sus decisiones didácticas e inscribiendo en sus intencionalidades educativas, sus expectativas, el deseo que moviliza el propio hacer docente, también la incertidumbre y la dificultad que viven, al pensarse enseñando con un grupo concreto. Se trata de un texto con carácter de borrador -siempre abierto y en elaboración- desarrollado en un espacio virtual que facilita el intercambio con el equipo docente de Práctica, en un ir y venir que busca orientar y acompañar el desarrollo de sus clases.

- Los autorregistros, escritos después de cada clase en sus diarios de formación, se centran en la reconstrucción de lo que (les) aconteció en el aula, en relación con el grupo y los conocimientos y saberes que pusieron en juego al enseñar. En este caso, buscamos que la escritura no quede en un plano descriptivo sino que, al poner en relación lo acontecido en cada clase con el guion conjetural previamente escrito, puedan repensar sus decisiones didácticas para el siguiente encuentro y también reflexionar, haciendo lugar a las tensiones que viven entre lo que desean ser y hacer como docentes y entre lo que pasa y les pasa en el encuentro con el grupo.

Los tres recursos narrativos descritos brevemente aquí, acompañan y recogen el recorrido que cada estudiante-practicante realiza en esta asignatura y constituyen una fuente de intercambio y conversación con quienes acompañamos ese recorrido como docentes de práctica. Asimismo, constituyen una fuente de consulta necesaria para elaborar el relato de experiencia que solicitamos al finalizar el curso donde, haciendo 
una mirada retrospectiva sobre lo vivido en la escuela, reflexionan y profundizan pedagógicamente (Contreras y Quiles, 2017) en aquello que la experiencia vivida (les) abre a pensar sobre el oficio de enseñar ${ }^{1}$.

\section{Análisis}

\subsection{Escritura y experiencia: hacia una relación narrativa con la formación}

La escritura configura un espacio que inscribe la experiencia (Sardi, 2017) y al ponerla en palabras, la hace visible y legible, tornándola pensable en tanto algo que nos sucede y afecta. Así lo expresan dos estudiantes ${ }^{2}$ en sus diarios de formación:

[Al escribir] pude ponerle palabras concretas a mucho de lo que estaba sintiendo y pensando, revisando y cambiando con respecto a mis concepciones sobre lo que implica la educación como fenómeno social, mi lugar y el de otros como estudiantes, (...) así como también mi lugar y el de otros como docentes. (Amalia)

Cuando era alumna de secundaria pensaba que los profesores la tenían fácil. (...) Ahora estoy del otro lado, en donde puedo ver las dificultades del quehacer docente dentro y fuera del aula (...) Por suerte las materias que estoy cursando en el cuatrimestre me dan bastantes herramientas para cuando esté frente a un grupo de alumnos (de eso se trata, ¿No?). (...) me permiten pensar, imaginar, visualizar cómo sería el momento en que esas actividades fueran dadas a un grupo de alumnos. Al fin y al cabo, es ahí donde nos vamos construyendo como profesores ¿No? (Helena)

Las palabras de ambas señalan el carácter mediador de esta escritura y dejan traslucir su textura subjetiva y relacional, evidenciando cómo la formación se va tejiendo con las propias vivencias, sentimientos e inquietudes, con lo que el otro y lo otro (les) provoca. Evidentemente, el yo que narra no registra de manera indiferente lo que pasa y le pasa en relación a las prácticas, sino que se detiene en aquello que afecta su ser estudiante-docente en formación, su saber y sus posibilidades de aprender.

A través de la escritura también se tornan visibles los lugares que habitan y el modo en que estos configuran la experiencia de formación: hay espacios que brindan herramientas para después, como indica Helena, y espacios de construcción de sí como docente, repletos de incertidumbres que interpelan la relación consigo misma. Como señala Amalia:

No sé si seré capaz de desempeñarme como deseo, no sé si el grupo escolar con el que me toque trabajar estará dispuesto a conectar con mis planificaciones; no sé hasta qué punto estoy ‘lista’ para ejercer la docencia.

O Nina cuando se pregunta:

¿Cómo me desenvolveré SOLA frente a un grupo? ¿Podré ganar su confianza? ¿Podré transmitir lo que sé? ¿Sé lo suficiente? ¿Podré manejar los tiempos en la clase? ¿Y los de la práctica con relación al resto de la cursada?

La escritura habilita una relación narrativa con las vivencias de la formación articulando el saber y el aprender en este espacio-tiempo con la propia historia personal. Asimismo, al escribir van cartografiando el terreno que habitan de manera singular, dando cuenta de la escisión que viven entre los tiempos y las exigencias de la práctica y los otros tiempos y exigencias académicas de la universidad; confrontando, también, el saber que tienen y el que necesitan al pensarse en el papel de profesoras que deben asumir. $\mathrm{Al}$ respecto Nina expresa:

(...) uno de los problemas que más tenemos los estudiantes de letras: nos encontramos sin tiempo y espacio para leer y releer por placer, para explorar nuevos textos y llevar cosas diferentes, para construir y reconstruir nuestro propio canon. (...) Siento que la conexión con el otro y con el saber mismo son cosas que quiero trabajar más a fondo como docente.

Podemos decir que, escribir en clave personal y reflexiva mueve a des-cubrir tensiones y a re-conocer los saberes que necesitan, aquellos que el oficio docente les reclama, no en términos instrumentales ni teóricos, sino vinculados a la relación educativa y a la propia presencia en ella, como sentidos que se labran en las prácticas, abriendo un camino de búsqueda personal de saber. Un camino que se elabora en primera persona, pero siempre en relación con el otro y con lo otro, como deja entrever Amalia:

Noto cómo mis preocupaciones van mutando: en un principio estaba más preocupada por la disciplina en el aula, pero mientras más avanzo en la planificación me doy cuenta de que si lo que

\footnotetext{
${ }^{1}$ Este relato es publicado y compartido con practicantes de otros profesorados al ser presentados en los "Encuentros de practicantes y residentes" organizados desde hace seis años en la UNSL (PROICO 04-4116).

${ }^{2}$ Las estudiantes autorizaron la publicación de los fragmentos transcritos en este artículo bajo seudónimos.
} 
propongo resulta interesante, si los chicos se sienten involucrados, motivados y desafiados puede ser que esa energía desbordante se vuelque en el aprendizaje.

La experiencia de escribir también les ayuda a reconocer que "el oficio docente se hace con uno mismo, con lo que uno es y lleva incorporado" (Contreras, 2010, p. 64):

En mi infancia y buena parte de la adolescencia, fui una aprendiz muy temerosa y extremadamente tímida, y notaba que los docentes siempre mostraban cierta predilección por los estudiantes que eran extrovertidos o participativos, así que yo tampoco me sentía muy cómoda con ese tipo de vínculo tan selectivo (...) La educación que recibí (...) me hizo experimentar todo lo que yo no quiero repetir en mi vida ni con mis estudiantes en el futuro. (Amalia)

Asimismo, la escritura expone el proceso vivencial y experiencial que involucra la formación; un proceso opacado y colonizado por exigencias académicas que tienden a cancelar la implicación del sujeto en el propio aprendizaje, obturando sus búsquedas personales. En este sentido, Nina es contundente:

Creo que una de mis inseguridades como futura docente es también una inseguridad frente al objeto de estudio. Debido al ajetreo de la cursada, no he encontrado momentos para reflexionar y reconstruir todos los saberes y conocimientos que he acumulado: no he podido apropiármelos más allá de la materia. Creo que cuando una tiene cierta seguridad en lo que está diciendo y haciendo puede, a la vez, permitirse cuestionarlo y enfocarse en otros aspectos como es el contacto con el otro.

\subsection{Exploración de sí: la relación con el saber como inquietud}

En la relación narrativa que entablan con lo que van viviendo y pensando al escribir las prácticas, la mirada se abre a la dimensión biográfica del aprender y, en esa apertura que liga presente y pasado en un movimiento recursivo, se enfrentan con ciertos obstáculos que inauguran caminos para repensarse. Esto ocurre, por ejemplo, cuando la propia relación con el saber resulta interpelada:

Mi relación con el conocimiento durante la etapa previa a la universidad no fue muy gratificante porque aprendí repitiendo de memoria (...), sentía que lo que me enseñaban no era significativo, la escuela en sí misma no tenía otro sentido más que el de terminarla. (Amalia)

Esta semana [nos pidieron] pensar una actividad para trabajar los puntos que pudimos rescatar del texto, pero sin recurrir a la explicación/exposición (...) Me di cuenta de lo mucho que me apoyo en la explicación de los textos y sobre todo, en la memorización. Me cuesta mucho poder salir de los márgenes que marca y poder relacionarlos con el debate que se da. Quizás por ello mis intervenciones en clase son mínimas. Es algo que debo seguir trabajando. (Nina)

La relación con el saber, dice Diker, "no designa el dominio de un saber específico, sino más bien, el modo en que un sujeto se posiciona frente al saber, a sus posibilidades de aprender y de hacer, en tanto individuos con deseos y anhelos de saber, de entender el mundo y entenderse en él" (2007, p. 227). Y esto es algo que al narrar(se) en las prácticas registran en clave de inquietud, abriendo a su reelaboración desde la experiencia. Ese movimiento que la escritura capta y hospeda, es sentido que se abre: "apertura de sí, una manera de pensarse y entenderse, en relación" (Contreras, 2007, p. 2); planteando -en el caso de las prácticasla doble exigencia de reposicionarse tanto frente al aprender como al enseñar. Además, observamos que en el guion conjetural y en los autorregistros van quedando huellas de la dificultad que este reposicionarse implica en la enseñanza y también el rastro del desencuentro que viven entre lo que desean y lo que hacen en sus prácticas:

En general, la clase de ayer salió como la planifiqué en el guion. Pude manejar bien el tiempo para dar y explicar todos los conceptos que abarcó el tema. Sin embargo, yo -como profesora- no quedé conforme con la clase presentada. Fue una clase muy expositiva, con mucha teoría para dar y no estoy segura de que las estudiantes hayan comprendido todo. (Nina)

\section{Conclusiones}

La enseñanza es un oficio delicado, "no sólo porque tratamos con otras personas, sino también, porque tratamos con nosotros mismos" (Contreras, 2013, p. 14); por eso, en toda situación educativa no sólo está en juego una mirada que se dirige hacia afuera, ligada al hacer, a lo que nos preocupa como docentes, sino también una mirada hacia adentro, atenta a lo que esa situación y el propio hacer en ella nos significa y plantea, lo que nos provoca, el modo en que nos afecta.

Ambas miradas, pero fundamentalmente la segunda, es lo que pretendemos captar y acoger al proponer la escritura en las prácticas como mediación que aspira a cultivar una relación fructífera entre experiencia y 
saber en la formación. Buscamos promover una escritura personal-reflexiva que constituya una práctica de exploración de sí y de lo otro, entendiendo que esta abre la posibilidad de narrar la experiencia y de hacer experiencia pues, al poner en relación el propio hacer, ser, saber, pensar y vivir, es posible que algo le suceda a quien escribe: algo que interpele su mirada, su atención, su imaginación, su relación personal con el mundo, abriéndola a nuevas posibilidades.

En este artículo exploramos esta potencialidad y el valor formativo que atribuimos a la escritura en las prácticas analizando textos elaborados por estudiantes-practicantes; $y$ advertimos que la escritura personalreflexiva posibilita desarrollar una relación narrativa con lo que viven en la formación, creando un espacio de exploración de sí, del propio ser y hacer como estudiante, que conecta con la historia personal, también con la identidad y el saber docente en construcción. Al narrar(se) en las prácticas, quien escribe se piensa en situación, piensa la propia presencia -en su dimensión biográfica y subjetiva- y expresa las tensiones que la configuran en el proceso formativo. En este sentido, la escritura trasluce lo que hay en juego en la formación como "proceso vivencial y experiencial" (Blanco, et al., 2016, p. 235), es decir, su materialidad como hacer concreto, mostrando sus dificultades y problemas, sus tensiones, pero sobre todo, su singularidad, puesta de manifiesto en la vitalidad, la búsqueda y orientación personal, las ganas de crecer, de ser cada una, cada uno, a su manera (Contreras, 2013, p. 3).

Así pudimos verlo en los fragmentos que transcribimos, donde la tensión entre el sentido personal de aprender y las exigencias académicas (propias de la universidad) queda al descubierto, expresándose en la vivencia escindida de sí como estudiantes-practicantes, en el cuestionamiento de la propia relación con el saber y en la tensión entre el saber que tienen y el que necesitan -porque el oficio docente se los reclama- al pensarse y vivirse como profesoras en las prácticas. Este movimiento que la escritura provoca es apertura a la experiencia, esto es, a la transformación de la propia relación con el otro, con lo otro y consigo mismo; un movimiento en el que cada una va encontrando sentidos nuevos para orientarse en el aprender y en el saber, en clave personal y en relación a la propia identidad docente en construcción; porque, la escritura personal-reflexiva recoge "lo que la identidad crea en tanto proceso: un conjunto de pensamientos acerca de uno mismo que es a la vez, narración, lo que nos hace decir quiénes somos y quiénes queremos ser, cómo nos proyectamos en alguien que es y va siendo a la vez, inacabadamente, que no está aquí todavía, que está por ser" (Nicastro y Greco, 2009, p. 60).

Para finalizar, nos interesa reflexionar un momento sobre las dificultades que la escritura como mediación en la formación (nos) plantea y sobre el extrañamiento y/o resistencias que suele provocar en las y los estudiantes. Indudablemente, se trata de una tarea compleja y espinosa: al pedirles que escriban en primera persona lo que hacen y viven en las prácticas, estamos pidiendo que se desplacen del "deber ser" al "estar ahí", en el propio proceso de formación (Blanco, et al., 2016, p. 233), exponiéndose al hacer "el ejercicio de desplazar la atención de aquello que está fuera de míp" para indagar en la relación que establecen con eso que les llega (p. 237). Este desplazamiento o pasaje del deber ser, que ocupa el ser con sentidos dados y que tiende a cancelar la interpretación de sí y desde sí de experiencias fundamentales como la de ser y la de saber, es lo que Blanco et al. (2016) denominan un "movimiento de la presencia". Y es eso lo que les pedimos cuando proponemos escribir en primera persona: una práctica compleja y conflictiva "en un contexto institucional como la universidad que tiende a negar, a olvidar y/o a cancelar la implicación del sujeto en el propio proceso de aprendizaje, provocando esa extrañeza que causa la ruptura entre conocimiento y vida" (p. 236).

Podemos decir entonces que, lo que está en juego al proponer la escritura personal-reflexiva como mediación en la formación, es el intento de "restablecer ese vínculo que reconoce el proceso generativo y relacional del saber" (Ellsworth, 2005, p. 235), asumiendo que el saber que el oficio docente requiere es siempre un proceso inconcluso, abierto y narrativo que se hace en la experiencia, mientras se aprende y no antes.

\section{Apoyos}

Laura Pañagua Domínguez se encuentra realizando la tesis doctoral bajo la concesión de una beca pre-doctoral, financiada por el Ministerio de Educación, Cultura y Deporte del Gobierno de España (FPU17/04219).

\section{Referencias}

Alliaud, A. (2017). Los artesanos de la enseñanz̨. Acerca de la formación de maestros con oficio. Paidós.

Alvarado, M. y Cortés, M. (2001). La escritura en la Universidad. Repetir o transformar. Lulú Coquette. Revista de Didáctica de la Lengua y la Literatura, (1), 19-23.

Biesta, G. (2017). El bello riesgo de educar. SM. 
Blake, C. (2013). Tensiones en las prácticas de escrituras escolares. En V. Sardi (Coord.), Relatos inesperados. La escritura de incidentes críticos en la formación docente en Letras (pp. 33-55). Universidad de La Plata.

Blanco, N., Molina, M. y Arbiol, C. (2016). Explorar las tensiones para transitar el abismo: buscando el sentido del oficio docente. En J. Contreras. (Coord.), Tensiones fructiferas: explorando el saber pedagógico en la formación del profesorado. Una mirada desde la experiencia (pp. 223-256). Octaedro

Bombini, G. (Coord.) (2012). Escribir la metamorfosis. Escritura y formación docente. El Hacedor.

Bombini, G. y Labeur, P. (2013). Escritura en la formación docente: los géneros de la práctica. Enunciación, 18(1), 19-29.

Carlino, P. (2005). Escribir, leer, y aprender en la universidad. Una introducción a la alfabetización académica. FCE.

Cifali, M. (2010). Enfoque clínico, formación y escritura. En L. Paquay (Coord.), La formación profesional del maestro. Estrategias y competencias (pp. 170-196). FCE.

Clandinin, J.D. (2013). Engaging in Narrative Inquiry. Left Coast Press.

Connelly, F. y Clandinin, J.D. (1988). Teachers as curriculum planners: Narratives of experience. Teachers College Press.

Contreras, J. (2007). Personalizar la relación. Aperturas pedagógicas para personalizar la enseñanza. Organización y Gestión Educativa, 15(4), 17-24.

Contreras, J. (2010). Ser y saber en la formación didáctica del profesorado: una visión personal. REIFOP, 24(2), 61-82.

Contreras, J. (2011). El lugar de la experiencia. Cuadernos de Pedagogía, 417, 60-63.

Contreras, J. (2013). Experiencia, escritura y deliberación: Explorando caminos de libertad en la formación didáctica del profesorado. Inter-Ação, 38(1), 1-35.

Contreras, J. (2016). Relatos de experiencia, en busca de un saber pedagógico. Revista Brasileira de Pesquisa (Auto) Biográfica, 01, 14-30.

Contreras, J. y Quiles-Fernández, E. (2017). Vivir y profundizar experiencias de enseñanza desde una perspectiva narrativa. En J. Contreras (Coord.), Enseñar tejiendo relaciones: una aproximación narrativa a los docentes y a sus clases de Educación Infantily Primaria (pp. 19-33). Morata.

Contreras, J., Quiles, E. y Paredes, A. (2019). Una pedagogía narrativa para la formación del profesorado. Márgenes, Revista de Educación de la Universidad de Málaga, O(0), 58-75. DOI:10.24310/mgnmar.v0i0.6624

Delory-Momberger, C. (2014). Experiencia y formación. Biografización, biograficidad y heterobiografía. Revista Mexicana de Investigación Educativa, 19(62), 695-710.

Diker, G. (2007). ¿Es posible promover otra relación con el saber? Reflexiones en torno del proyecto DAS. En R. Baquero, G. Diker y G. Frigerio (Comps.), Las formas de lo escolar (pp. 225-244). Del Estante Editorial.

Ellsworth, E. (2005). Posiciones en la enseñanza. Diferencia, pedagogía y el poder de la direccionalidad. Akal.

Molina, M., Blanco, N. y Arbiol, C. (2016). Dejarse tocar para que algo nos suceda. En J. Contreras (Comp.), Tensiones Fructiferas. Explorando el saber pedagógico en la formación del profesorado (pp. 111-150). Octaedro.

Mortari, L. (2002) Tras las huellas de un saber. En Diótima, El perfume de la maestra. En los laboratorios de la vida cotidiana (pp. 153-162). Icaria.

Mortari, L. (2012). Learning thoughtful reflection in teacher education. Teachers and Teaching, 18(5), 525-545. DOI: $10.1080 / 13540602.2012 .709729$

Nicastro, S. y Greco, B. (2009). Entre trayectorias. Escenas y pensamientos en espacios de formación. Homo Sapiens.

Pañagua, L., Martín Alonso, D. y Blanco, N. (2019). Escritura reflexiva y desarrollo de saberes experienciales. Tensiones y posibilidades. REIFOP, 94(3), 11-28.

Pérez Gómez, A. (2010). La naturaleza del conocimiento práctico y sus implicaciones en la formación del profesorado. Infancia y Aprendizaje, 33(2), 171-177.

Sardi, V. (2013). La escritura de las prácticas en la formación docente en Letras. En V. Sardi. (Coord.), Relatos inesperados. La escritura de incidentes críticos en la formación docente en Letras (pp. 10-32). Universidad de La Plata.

Sardi, V. (2017). Escribir la práctica, inscribir la experiencia. Saberes y prácticas. Revista de Filosofía y Educación, 2. Recuperado de: saberesypracticas.uncu.edu.ar

Serrano, S. (2014). La lectura, la escritura y el pensamiento. Función epistémica e implicaciones pedagógicas. Lenguaje, 42(1), 97-122.

Sierra, J.E., Caparrós, E., Molina, M. y Blanco, N. (2017). Aprender a través de la escritura. Los diarios de prácticas y el desarrollo de saberes experienciales. Revista Complutense de Educación, 28(3), 673-688. DOI:0.5209/rev_RCED.2017.v28.n3.49708

Sofías (2007). Saber es un placer. La práctica política de mujeres que buscan dar sentido libre a la educación. Horas y HORAS.

Souto, M. (2016). Pliegues de la formación. Sentidos y herramientas para la formación docente. Homo Sapiens. 
Tardiff, M. (2004). Los saberes del docente y su desarrollo profesional. Madrid: Morata.

Van Manen, M. (2015). Pedagogical tact: knowing what to do when you don't know what to do. Left Coast Press. 